\title{
La formación en competencias de la biblioteca de la Universidad de La Laguna en el contexto de la cooperación universitaria
}

\author{
Carmen Julia Hernández ${ }^{\star}$, José Manuel Erbez ${ }^{\star \star}$
}

Resumen: Se recoge la experiencia de La Biblioteca de la Universidad de La Laguna desde el curso 2006-2007, en el que comienza a cambiar su modelo de formación de usuarios por otro basado en formación en competencias, en consonancia con los requisitos que el nuevo entorno educativo recogía. Se analiza lo que ha significado dicha transformación desde el punto de vista de la planificación de un nuevo programa formativo y la adquisición de nuevas competencias por parte de los bibliotecarios para ayudar a los usuarios a conseguir unas destrezas, habilidades y conductas transferibles a su vida académica y personal, y a su futuro laboral en relación con la gestión de información. También se aborda el significado de trabajar de forma cooperativa con otras bibliotecas universitarias, como resultado de la firma de un convenio en el año 2010 con el objetivo de compartir nuestro modelo de formación para alumnos de nuevo ingreso y evaluarlo mediante la comparación entre las distintas universidades. Finalmente, se trata la colaboración entre bibliotecarios y profesores para la inclusión de la formación en competencias informacionales en las guías docentes.

Palabras clave: competencias informacionales, alfabetización informacional, formación, cursos virtuales, eLearning., cooperación bibliotecaria, ALFIN, UNICI2.

\section{Competence-based training at the Universidad de la Laguna's library in the context of inter-university cooperation}

Abstract: This paper deals with the experience of the Library of the University of La Laguna since 2006-2007, when it began to change its model of user training for a new one based on information competences, in line with the requirements of the new European Higher Education Area. It analyzes what this change has meant from the point of view of planning a new training program and the acquisition of new competences by the librarian staff in order to belp users acquire information management skills, abilities and behaviours that can be applied in their academic, professional and personal lives. It also describes cooperation with other university libraries, in the framework of an agreement signed in 2010. The aim of this agreement is for our model to be shared for training new students and subsequently evaluating it through a comparison of results from the different universities. Finally, it deals with the collaboration between librarians and professors for including information competence training in course guides.

* Universidad de La Laguna, Biblioteca.

Correo-e: cjhdez@ull.edu.es

** Universidad de La Laguna, ETSI Informática.

Correo-e: jerbez@ull.edu.es

Recibido: 31-01-2012; 2. ${ }^{\text {a }}$ versión: 14-02-2012; aceptado: 26-03-2012. 
Keywords: information literacy, information competences, user training, eLearning, $l i$ brary cooperation, UNICI2.

\section{La formación en competencias de la Biblioteca de la ULL: un trabajo conjunto en nuestra Universidad}

\subsection{El camino recorrido}

Hasta el año 2005 la actividad formativa desarrollada en la Biblioteca de la ULL consistía en la tradicional formación de usuarios, basada en ofrecerles una serie de sesiones presenciales sobre los servicios, recursos y herramientas disponibles, y sobre todo sobre el funcionamiento del catálogo y de determinadas bases de datos. El alcance de esta actividad era muy desigual, dado que ni se ofertaba del mismo modo en todos los puntos del servicio (dependiendo en gran medida tanto de la disponibilidad como de la actitud del personal) ni la respuesta de los usuarios era la misma (por ejemplo, los de Humanidades solían ser más receptivos que los de Ciencias). Por lo tanto, el número de alumnos a los que se llegaba era muy limitado, con cifras que se mantenían a lo largo del tiempo.

Tras analizar esta cuestión detenidamente, y probar diferentes fórmulas para mejorar estos resultados (campañas de difusión, elaboración de materiales formativos, llamamientos a una mayor implicación del personal y el profesorado...) se llegó a la conclusión de que la formación presencial tenía inconvenientes insalvables: la dificultad de reunir a los usuarios en un espacio físico y en un horario determinado, con los problemas de interferencia con el horario de clases, disponibilidad de espacios y medios técnicos, compatibilidad con las otras tareas a desarrollar por los bibliotecarios... Todo ello hacía que no pudiéramos aspirar a llegar más que a una pequeña parte de nuestros usuarios, y a costa de invertir muchas horas de trabajo.

Una vez que la Biblioteca quiso abordar el cambio de orientación en la formación de usuarios, hicimos un análisis sobre nuestras debilidades, amenazas, fortalezas y oportunidades para organizar un nuevo servicio de formación en competencias e investigamos lo que en nuestra Universidad se estaba haciendo dentro de los planes de estudio para incorporar las competencias básicas transversales.

Desde el año 2006 la Biblioteca, debido a las conclusiones a las que habíamos llegado sobre nuestra experiencia formativa, y ante el reto del Espacio Europeo de Educación Superior (EEES) y el cambio de concepción del modelo de enseñanza-aprendizaje, comenzó a adaptar su programa de formación.

En su Plan estratégico 2006-2008 definió una línea de trabajo sobre la formación en competencias informacionales como apoyo al nuevo modelo educativo.

En esos primeros momentos de cambio nos planteamos la necesidad de alfabetizar en información, lo que conllevaba dotarnos de un programa de formación que ayudara al alumnado, no solo a ser capaz de usar recursos de información 
accesibles desde la biblioteca y conocer sus servicios, sino también a localizar información de calidad entre la gran cantidad de recursos existentes en diferentes soportes, evaluarla, usarla de manera ética y comunicarla de forma adecuada. Además, debía contribuir a que adquiriera un método de trabajo más autónomo, fuera responsable de la creación del propio conocimiento y de trasladar las competencias adquiridas a la actividad académica, personal y laboral, en el contexto de un aprendizaje continuo.

La Biblioteca se planteó apoyar el nuevo modelo de formación a través de aulas virtuales, en la plataforma de enseñanza/aprendizaje Moodle, aprovechando la creación en la Universidad de un Campus Virtual y una Unidad de Docencia Virtual encargada de gestionarlo. La buena relación de nuestro Servicio con los responsables de dicha Unidad ayudó a que aprovecháramos la nueva experiencia de $e$-learning que se estaba desarrollando en nuestra Universidad. La Unidad puso a nuestra disposición toda una sección del Campus Virtual con plena libertad para crear, modificar, organizar y suprimir aulas, lo que facilitó mucho el trabajo.

La Biblioteca vio en este nuevo recurso una oportunidad para reorientar su actividad formativa, aprovechando las posibilidades de la docencia virtual para superar las barreras que nos impedían dar una formación de mejor calidad, basada en competencias y dirigida a un gran número de usuarios.

La gran ventaja que para los objetivos de la Biblioteca ofrecía la formación virtual era la oportunidad de liberarse de la limitación espacio-temporal. A ello se añadía la posibilidad de emplear materiales formativos en distintos formatos (texto, presentaciones, vídeos...) y programar tareas que permitieran al usuario practicar los conocimientos impartidos y al tutor evaluar la adquisición de dichos conocimientos.

El nuevo modelo de formación virtual se complementaba con alguna actividad presencial para aprovechar también las ventajas de la comunicación directa con el usuario.

Una parte de la actividad formativa de nuestra Biblioteca ha sido recogida en el monográfico sobre Competencias informacionales y digitales en la educación superior (2010) de la Revista de Universidad y Sociedad del Conocimiento (Hernández, 2010) y en Veintitantas experiencias ALFIN y una canción esperanzada (Hernández, 2011).

Desde que comenzamos a formar en competencias informacionales, en el curso académico 2006-2007, hemos recorrido un largo camino que, aunque exitoso, no ha estado exento de problemas:

- El temprano interés mostrado por nuestro Servicio por trabajar en formación en competencias, anticipándose en buena medida al resto de la Universidad.

- La resistencia inicial de algunos bibliotecarios a asumir el nuevo rol de formador en competencias y, en algunos casos, compatibilizarlo con el resto de sus tareas. 
- La falta de interés de gran parte del profesorado, en los primeros momentos de implantación de los cursos, por temas relacionados con la adquisición de competencias, en un período de transición al nuevo modelo educativo.

- Las inercias existentes en la Universidad para asumir los cambios propuestos por la Biblioteca, especialmente en lo relacionado con la integración de la formación en competencias dentro de toda la actividad docente.

\subsection{El Plan de Formación CI2}

A lo largo de estos años nos hemos dotado de dos planes de formación, el inicial, un Programa de Formación ALFIN, y el actual Plan de Formación CI2 que integra cursos dirigidos a la comunidad universitaria donde se incluyen competencias informacionales e informáticas (2012). La inclusión de estas últimas ha sido posible gracias a la implicación del Vicerrectorado TIC de nuestra Universidad. Su participación se centra en el apoyo de la formación en competencias informáticas en el curso de tres créditos ECTS (como complemento de formación) dirigida a alumnado de segundo y tercero de grado.

El actual plan contempla para el alumnado tres niveles obligatorios de formación (primer curso, cuarto y posgrado) y uno voluntario (complemento de formación de tres créditos ECTS).

El plan integra formación para el Personal Docente e Investigador con el objetivo de colaborar en su reciclaje de competencias informacionales y concienciarle de la importancia de trabajarlas en las asignaturas. E incluye formación para el personal de la Biblioteca y usuarios externos que necesiten reciclar competencias informacionales.

Establece quiénes son los responsables de la formación, el tipo de cursos a implementar y los objetivos de trabajo de los diferentes grupos (uno por cada tipo de curso) responsables de la aplicación del programa. Contempla también cómo se debe incorporar la formación en las titulaciones de grado y posgrado, siempre como actividad dentro de la guía docente de las asignaturas obligatorias en los diferentes niveles de aprendizaje.

También contempla la forma de evaluar los cursos, el aprendizaje de los usuarios y el grado de satisfacción de alumnos, profesorado y bibliotecarios, así como el modelo de certificación que se ofrece a los diferentes tipos de usuarios, a los formadores y al profesorado colaborador.

El plan, además, recoge cómo difundir nuestras acciones formativas. Entre las acciones desarrolladas en este sentido están la elaboración de un video promocional (http://youtu.be/R1JiJHa3NJA) con la participación de profesores, alumnos y bibliotecarios para difundir en red la importancia de la adquisición de las competencias informacionales, y la edición de un tríptico informativo dirigido a la concienciación del profesorado sobre la formación en competencias.

En el siguiente cuadro se puede apreciar la oferta formativa que incluye nuestro plan (tabla I). 


\section{TABLA I}

\section{Plan de formación de la Biblioteca}

\begin{tabular}{|c|c|c|c|c|c|}
\hline \multicolumn{2}{|c|}{ Usuarios } & $\begin{array}{c}\text { Formación } \\
\text { en competencias } \\
\text { en información }\end{array}$ & Certificación & Nivel & Horas \\
\hline \multirow{3}{*}{ Grado } & $10^{\circ}$ & $\begin{array}{l}\text { Formación inicial } \\
\text { - Jornadas de bienveni- } \\
\text { da ( } 1,5 \text { h. presencial). } \\
\text { - Visita guiada ( } 1 \text { hora). } \\
\text { - Curso básico sobre } \\
\text { competencias en infor- } \\
\text { mación ( } 10 \text { horas vir- } \\
\text { tuales de trabajo autó- } \\
\text { nomo). } \\
\end{array}$ & $\begin{array}{l}\text { Vicerrectorado } \\
\text { Servicios } \\
\text { Universitarios }\end{array}$ & Iniciación & 12,5 horas \\
\hline & $2 .^{\circ} / 3 .^{\circ}$ & $\begin{array}{l}\text { Formación intermedia } \\
\text { Curso intermedio sobre } \\
\text { competencias en infor- } \\
\text { mación e informáticas } \\
\text { (complementos a la for- } \\
\text { mación). }\end{array}$ & Acreditado & Especialización & $\begin{array}{l}3 \text { créditos } \\
\text { ECTS }\end{array}$ \\
\hline & $4 .^{\circ}$ & $\begin{array}{l}\text { Formación avanzada } \\
\text { Curso de formación en la } \\
\text { asignatura de preparación } \\
\text { del proyecto fin de grado. }\end{array}$ & $\begin{array}{l}\text { Vicerrectorado } \\
\text { Servicios } \\
\text { Universitarios }\end{array}$ & Avanzado & 15-20 horas \\
\hline \multicolumn{2}{|c|}{ Posgrado } & $\begin{array}{l}\text { Formación avanzada } \\
\text { Curso para el desarrollo } \\
\text { del trabajo de master y } \\
\text { doctorado. }\end{array}$ & $\begin{array}{l}\text { Vicerrectorado } \\
\text { Servicios } \\
\text { Universitarios }\end{array}$ & Reciclaje & 15 horas \\
\hline \multicolumn{2}{|l|}{ PDI } & $\begin{array}{l}\text { Formación } \\
\text { para el reciclaje }\end{array}$ & $\begin{array}{l}\text { Vicerrectorado } \\
\text { Calidad }\end{array}$ & Reciclaje & 20 horas \\
\hline \multicolumn{2}{|c|}{ PAS Biblioteca } & $\begin{array}{l}\text { Formación } \\
\text { para el reciclaje }\end{array}$ & Gerencia & Reciclaje & 10 horas \\
\hline \multicolumn{2}{|c|}{ Externos } & $\begin{array}{l}\text { Formación } \\
\text { para el reciclaje } \\
\text { Curso de formación para } \\
\text { profesionales de la infor- } \\
\text { mación. }\end{array}$ & $\begin{array}{l}\text { Certificada } \\
\text { Vicerrectorado } \\
\text { Servicios } \\
\text { Universitarios }\end{array}$ & Reciclaje & 20 horas \\
\hline
\end{tabular}

Nuestro actual Plan de Formación se ha dotado de una imagen y marca, "Aula CI2", que define bien nuestro objetivo: formar al alumnado universitario en dos competencias básicas transversales, a través de aulas virtuales en una línea de cooperación entre servicios universitarios (Servicio TIC, la Unidad de Docencia Virtual y Biblioteca). 


\section{FIGURA 1}

\section{Logo del plan de formación}

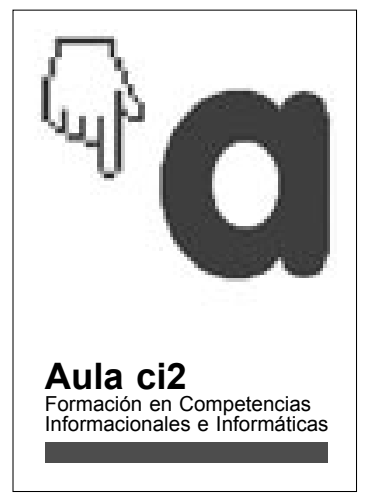

Para trabajar de manera eficaz se creó una Comisión de Formación de la Biblioteca formada por cinco personas: la Subdirectora de Servicios y Personal como coordinadora general y los coordinadores de los cuatro equipos de trabajo, cada uno dedicado a la organización de la formación de un tipo de usuario: alumnos de grado, alumnos de posgrado y personal docente e investigador (PDI), personal de administración y servicios (PAS) y usuarios externos.

\subsection{El trabajo conjunto en nuestra Universidad para formar en competencias}

La búsqueda de aliados internos (vicerrectores, decanos, coordinadores de calidad, profesores y otros servicios) siempre ha sido importante para la Biblioteca. El camino no ha sido fácil, quizás porque fuimos una de la primeras bibliotecas universitarias en implementar la formación en competencias informacionales. Además, en los primeros momentos nuestra Universidad estaba más preocupada por la puesta en práctica de la normativa relacionada con el nuevo espacio de educación superior que por atender a estas cuestiones.

Para la integración académica de la formación que ofrece la Biblioteca se cuenta con el apoyo del Vicerrectorado de Servicios Universitarios, así como de los Vicerrectorados responsables de calidad e innovación educativa y TIC. En estos últimos meses estamos trabajando con el Vicerrectorado TIC para implementar la formación en competencias informáticas en los cursos para alumnado de primero de grado.

Desde un primer momento se pensó que fuera un proyecto de cooperación entre profesores y bibliotecarios, aprovechando la estrecha relación que ambos colectivos han mantenido siempre en nuestra universidad. Comenzamos buscando aliados con los de primer curso, obteniendo inicialmente el apoyo de unos pocos, para ir sumando aliados con el paso del tiempo. 
Como la colaboración sigue siendo voluntaria, los bibliotecarios, antes de iniciarse cada curso académico, deben contactar con el profesorado de primer curso que esté dispuesto a incluir esta actividad en la guía docente. En los primeros momentos el éxito de esta búsqueda dependía de las buenas relaciones personales del bibliotecario con algún profesor. Muchos profesores que veían con buenos ojos el proyecto temían que el hecho de que sus alumnos realizaran el curso les restara tiempo para seguir la asignatura, y no hay que descartar que algunos vieran esta iniciativa de la Biblioteca como una intromisión en sus competencias docentes. Sin embargo, gracias a los buenos resultados de los cursos y la satisfacción de los primeros profesores colaboradores con la experiencia, así como a una importante labor de difusión realizada por distintos medios (elaboración de un folleto informativo, reuniones con decanos, directores y coordinadores de calidad de los centros, contacto directo con el profesorado) se ha conseguido ir concienciando a los docentes de que esta iniciativa supone un importante apoyo en su labor, dado que proporciona al alumno herramientas para obtener un mayor rendimiento en sus estudios. El éxito en este terreno se refleja en el hecho de que cada vez son más los profesores que colaboran con este programa y lo incluyen en la guía docente de la asignatura.

Si inicialmente, en el curso 2006-2007, contamos con 10 profesores de asignaturas troncales de primer año de carrera, en ocho titulaciones, en este curso académico 2011-2012, contamos con 44 profesores en 41 titulaciones de grado (de 42 títulos de grado existentes en nuestra Universidad), en asignaturas obligatorias.

En la siguiente tabla II se puede apreciar cómo ha ido evolucionando favorablemente el número de formadores y profesorado implicado en el programa de formación de la Biblioteca.

TABLA II

Evolución de la formación

\begin{tabular}{l|r|r|r|r|r|r}
\hline $\begin{array}{c}\text { Formación en competencias: } \\
\text { cursos virtuales }\end{array}$ & $\begin{array}{c}\mathbf{2 0 0 6 -} \\
\mathbf{2 0 0 7}\end{array}$ & $\begin{array}{c}\mathbf{2 0 0 7 -} \\
\mathbf{2 0 0 8}\end{array}$ & $\begin{array}{c}\mathbf{2 0 0 8 -} \\
\mathbf{2 0 0 9}\end{array}$ & $\begin{array}{c}\mathbf{2 0 0 9 -} \\
\mathbf{2 0 1 0}\end{array}$ & $\begin{array}{c}\mathbf{2 0 1 0 -} \\
\mathbf{2 0 1 1}\end{array}$ & $\begin{array}{c}\mathbf{2 0 1 1 -} \\
\mathbf{2 0 1 2}\end{array}$ \\
\hline Bibliotecarios formadores & 8 & 23 & 22 & 27 & 25 & $27^{*}$ \\
\hline Profesores colaboradores & 10 & 29 & 23 & 42 & 45 & 44 \\
\hline Titulaciones 8 & 18 & 18 & 36 & 36 & 41 & \\
\hline Cursos realizados & 9 & 35 & 24 & 34 & 42 & $47^{*}$ \\
\hline Alumnos matriculados & 292 & 1.103 & 944 & 1.710 & 3.441 & $3.020^{*}$ \\
\hline Alumnos aprobados & 204 & 719 & 836 & 1.303 & 2.886 & $2.747^{*}$ \\
\hline
\end{tabular}

Los datos con asterisco corresponden a los resultados obtenidos de la formación desarrollada sólo en el primer cuatrimestre 2011-2012. 
Tanto el Vicerrectorado del que dependemos, Servicios Universitarios, como el Vicerrectorado de Calidad e Innovación Educativa, han estado trabajando para que la formación que da la Biblioteca a los alumnos de grado (primer curso y cuarto curso) quede recogida en las guías docentes para tener evidencias de lo que se está realizando. Este curso académico ya es una realidad, de forma que en la mitad de las guías docentes de primero de los 41 títulos de grado existentes se ha recogido dicha formación, siendo del 100\% en las de cuarto curso.

Esto ha sido un gran logro, pues en la guía se incluye: los objetivos del curso de formación en competencias, el papel que realiza el bibliotecario formador, el número de horas que debe emplear el alumno, el porcentaje de valor en la asignatura, etcétera.

En cuanto a la búsqueda de aliados entre el profesorado del posgrado, ha sido más compleja; por el momento el número de profesores colaboradores y la inclusión en la guía docente es menor, comparativamente, que en el grado. Actualmente sólo estamos abordando la formación en másteres oficiales, por lo que nos queda trabajar con los títulos propios.

En 2009-2010, de un total de 24 másteres oficiales, se dio formación en 6, en el 2010-2011, de un total de 31 másteres, se formó en 9; y en el presente curso académico, de un total de 34 másteres, tenemos previsto dar 15 cursos, habiéndose impartido ya en 12 másteres en el primer cuatrimestre (tabla III).

TABLA III

Evolución de la formación para posgrado

\begin{tabular}{l|r|r|r}
\hline \multicolumn{1}{c|}{ Formación en competencias: cursos virtuales } & 2009-2010 & 2010-2011 & $\mathbf{2 0 1 1 - 2 0 1 2}$ \\
\hline Bibliotecarios formadores & 7 & 10 & 12 \\
\hline Profesores colaboradores & 7 & 7 & 11 \\
\hline Masters & 6 & 9 & 12 \\
\hline Alumnos matriculados & 134 & 151 & 261 \\
\hline Alumnos aprobados & 92 & 138 & 226 \\
\hline Incluidos en guía docente & 0 & 3 & 9 \\
\hline
\end{tabular}

Siempre ha habido un apoyo expreso del grupo de gobierno de nuestra Universidad a la labor formativa que ha estado haciendo la Biblioteca. El actual plan de gobierno (Universidad de La Laguna, 2011) incorpora la ampliación de la formación de usuarios en competencias informacionales e informáticas a los diferentes perfiles de usuarios. Este objetivo es responsabilidad del Vicerrectorado del que depende la Biblioteca. En las directrices presupuestarias de la Universidad para el año 2011 y en las del 2012 aparece consignado un apartado para el desarrollo de acciones relacionadas con competencias informacionales. En el presupuesto de la Biblioteca de estos años, se consignan las cantidades de 10.000 y 6.000 euros, respectivamente. 
Actualmente estamos trabajando para lograr un modelo de certificación que permita al alumno obtener un documento acreditativo de las competencias adquiridas. Ello nos ha obligado a trabajar de forma estrecha con responsables de nuestro Vicerrectorado y con la agencia certificadora.

\subsection{El papel del bibliotecario formador en la formación en competencias}

Nuestros bibliotecarios siempre se han dedicado a formar a usuarios, sobre todo en el manejo de herramientas (catálogo, bases de datos...), pero nos planteamos que había que cambiar algunos aspectos de la formación del personal para conseguir que fueran capaces de instruirles en competencias básicas transversales, especialmente las relacionadas con la gestión de la información.

En un contexto de cambio tecnológico en el que la producción de información crece a un ritmo exponencial, el papel del bibliotecario es importante, no sólo como intermediario que organiza y pone a disposición de los usuarios recursos de información en diferentes soportes y con formas de acceso múltiples, sino como formador que colabora con el aprendizaje de los estudiantes para ser competentes en la gestión de información.

Entendimos que si cambiaban las necesidades de nuestros usuarios, y era evidente que se iban a producir con las nuevas exigencias del proceso de Bolonia, teníamos que mejorar nuestras capacidades, aptitudes y conductas para formar de otra manera.

Dicho cambio no ha sido traumático, ya que nuestra profesión siempre ha estado en constante transformación. La figura del bibliotecario formador en competencias ha sido en nuestra Biblioteca un compromiso asumido sin grandes dificultades, aunque, como en todas las organizaciones, la nueva misión fue entendida de forma inmediata y decidida por unos y de forma más lenta y dubitativa por otros. Inicialmente algunos bibliotecarios entendían que impartir formación virtual no formaba parte de sus funciones como bibliotecario; incluso algunos llegaron a considerar que no se encontraban capacitados para realizar esta tarea. La primera reticencia fue más fácil de resolver, ya que se entendió pronto que no se trataba de asumir una nueva función, sino de realizar formación de otra manera. La otra cuestión resultaba más delicada, ya que muchas personas alegaban, no sin razón, que para poder impartir formación en competencias informacionales a través de aulas virtuales era preciso dominar esos temas; esta reticencia fue vencida gracias a la formación recibida sobre las competencias informacionales, el e-learning, la plataforma de enseñanza-aprendizaje Moodle, los aspectos didácticos, etc., así como al propio diseño de los cursos, pensado para minimizar todo lo posible las tareas de tutorización y evaluación.

En la actualidad, todos los bibliotecarios han asumido el nuevo rol y se implican decididamente en el aprendizaje de los usuarios. Han asumido nuevas 
competencias y son un elemento fundamental que colabora con el profesorado en la formación del alumnado.

Debido a la actitud positiva general de los bibliotecarios pudimos estructurar la mejora de los cursos dirigidos a los diferentes tipos de usuario, a través de grupos de trabajo específicos para cada tipo de curso. Los integrantes de dichos grupos están realizando una gran labor que hace posible que nuestro programa mejore y se difunda tanto dentro como fuera de nuestra Universidad.

Desde la dirección de la Biblioteca se ha propiciado tanto la formación de los bibliotecarios, a través de cursos organizados por la propia Biblioteca y del Plan de Formación del Personal de Administración y Servicios de la Universidad, como la participación en eventos (talleres ALFIN, jornadas CRAI, etc.) donde se debate el tema de la formación en competencias informacionales e informáticas y el papel que juega el bibliotecario.

La formación de los bibliotecarios se ha reforzado por medio de la plataforma Moodle, donde se han creado espacios de comunicación e intercambio de ideas.

Un aspecto que también ha contribuido a que nuestros bibliotecarios asumieran con facilidad el rol de formadores en competencias fue la difusión temprana de temas relacionados con la formación en competencias. Un representante de nuestra Biblioteca formó parte del grupo de REBIUN sobre ALFIN (http://rebiun.org/temasdetrabajo/alfin.html), y actualmente participa en el grupo de trabajo delegado de la Comisión intersectorial CRUE TIC y REBIUN para CI2 (http://ci2.es/). Nuestra participación en estos grupos de trabajo hizo que desde muy pronto se realizaran charlas informativas y formativas sobre estas cuestiones para los bibliotecarios.

Las nuevas funciones que debían asumir los bibliotecarios formadores fueron claramente reflejadas por González y Hernández (2007):

- Difundir acciones relacionadas con la formación en competencias.

- Formarse para mejorar su conocimiento sobre recursos de información.

- Preparar actividades para evaluar el aprendizaje de los usuarios.

- Conocer y utilizar métodos pedagógicos.

- Mejorar sus conocimientos tecnológicos.

- Utilizar un nuevo modelo de enseñanza/aprendizaje a través de aulas virtuales.

- Colaborar en la elaboración de materiales didácticos.

- Hacer comprender al profesorado la importancia de formar a los alumnos en competencias informacionales.

- Ayudar al profesorado en su reciclaje en formación en competencias informacionales

- Intercambiar experiencias con bibliotecarios de otras universidades. 


\subsection{El modelo de formación para alumnos de primero de grado}

Para la Biblioteca siempre ha sido un objetivo básico formar, fundamentalmente al alumnado de nuevo ingreso, pues accede a la Universidad con escasas competencias informacionales y con unas limitadas competencias informáticas. Por ello, el modelo de formación que se trabajó inicialmente es el dirigido a estos alumnos. Este modelo de curso es el que nos ha dado más visibilidad, por ser el que hemos presentado en diferentes eventos y cursos, así como el que ha conllevado la firma del convenio entre varias bibliotecas universitarias, que luego explicaremos.

El actual modelo de curso, fruto de un constante proceso de revisión y evaluación, tiene la siguiente estructura:

- Se comienza con un apartado inicial donde se explican los objetivos del curso, su organización y los criterios de evaluación; además, se incluye una breve encuesta destinada a conocer el perfil de los alumnos y un cuestionario con una batería de preguntas basadas en los contenidos del curso; el objetivo de este cuestionario es conocer qué nivel de conocimientos previos tiene el alumno, y compararlo con los resultados de un test similar que tienen que realizar al finalizar la formación.

- Con el tema 1 comienzan los contenidos propiamente dichos, abordando el proceso de búsqueda de información: qué es una fuente de información, aspectos a tener en cuenta antes de hacer una búsqueda, cómo valorar la información encontrada.

- El tema 2 trata sobre cómo localizar información en el catálogo de la Biblioteca y cómo interpretar un registro bibliográfico.

- El tema 3 aborda cómo localizar información electrónica a través de la web de la Biblioteca, y especialmente en PuntoQ, el portal de acceso y buscador de recursos electrónicos de la ULL.

- El tema 4 explica cómo localizar y evaluar información en Internet.

- El tema 5 pretende concienciar al alumno sobre la necesidad de evitar el plagio en la elaboración de trabajos académicos, y le explica cómo, cuándo y por qué citar la información encontrada, dando unas nociones generales sobre las citas bibliográficas y explicando alguno de los formatos de cita específicos (APA, ISO/UNE, Chicago, Vancouver, IEEE), elegido según cada titulación y de acuerdo con el profesor colaborador.

- El tema 6 explica el proceso de elaboración de un trabajo de clase: las partes que debe tener, los pasos que hay que seguir, consejos de presentación, etc. Además incluye algunos consejos prácticos para hacer una presentación con diapositivas, usando PowerPoint u otra herramienta similar.

- El tema 7 trata la web 2.0, haciendo hincapié en conceptos como la computación en la nube, las herramientas cooperativas (Google Docs, wikis, blogs, etc.) y las posibilidades que ofrecen las redes sociales para la vida académica y laboral. 
Se puede ver un ejemplo en la siguiente dirección: http://campusvirtual.ull.es/ formacion/course/view.php?id=551 (entrar como invitado).

Cada uno de estos temas incorpora unos breves textos explicativos, enriquecidos con esquemas, presentaciones, vídeos, etc., así como tutoriales en distintos formatos, la mayor parte realizados por la propia Biblioteca. Se complementan con una o varias tareas que permiten al alumno comprobar si ha entendido lo explicado y al tutor evaluar al alumno para certificar su aprovechamiento del curso. Estas tareas consisten en cuestionarios autoevaluables con varias preguntas en las que el alumno debe elegir la respuesta correcta entre varias alternativas. Otro elemento de los cursos son los foros, herramientas de comunicación entre el tutor y los alumnos y de éstos entre sí, y cuyo objetivo no es solo transmitir anuncios y novedades o resolver dudas y problemas, sino también contribuir al aprendizaje mediante el intercambio de opiniones. El curso se cierra con un cuestionario final cuyos resultados no solo permiten evaluar el nivel de adquisición de los contenidos por parte del alumno, sino también, por comparación con el test inicial, ver si se ha producido algún progreso con respecto al nivel que el alumno tenía previamente.

Por último, el curso incluye una encuesta de satisfacción que sirve para conocer qué aspectos del curso son mejor o peor valorados con vistas a su revisión y mejora.

Los cursos permanecen abiertos durante 10 días, en los que los alumnos son libres de entrar y trabajar en el momento que prefieran, lo que les permite adaptar el ritmo de realización del curso a su disponibilidad horaria. Generalmente, suelen emplear entre cinco y diez horas en total para completar el curso.

La calificación que obtienen los alumnos es la de "apto" o "no apto". Para aprobar es necesario que realicen todas las tareas, con una calificación media entre todas ellas superior a 5. Además, es obligatorio que participen en el foro al menos una vez a lo largo del curso. El tutor puede valorar también otras cuestiones, como el interés demostrado. Los alumnos que superan el curso obtienen un certificado de aprovechamiento avalado por el Vicerrectorado de Servicios Universitarios.

\subsection{El modelo de formación para alumnos de primero adaptado a otro tipo de usuarios}

Aunque los cursos dirigidos a alumnos de primero son los que ocupan la mayor parte de nuestra actividad, también estamos prestando atención a otros colectivos. Así, tenemos un modelo de curso dirigido a alumnos de posgrado, que se desarrolla en colaboración con los coordinadores de los diferentes másteres, y cuyos contenidos suponen básicamente una ampliación y profundización de los que se imparten a los alumnos de primero, haciendo hincapié en cuestiones como las búsquedas en bases de datos especializadas, la propiedad intelectual y los derechos de autor o los gestores de referencias bibliográficas. 
El modelo de curso de posgrado ha servido para diseñar otro dirigido a alumnos de cuarto curso de grado, con el objetivo de prepararles para la tarea de realizar el trabajo fin de grado necesario para obtener su titulación. Probablemente, conforme todos los alumnos de cuarto vayan realizando este curso, será menos necesario, quedando limitado a aquellos alumnos que por alguna circunstancia no hayan recibido esta formación anteriormente.

El modelo de posgrado también es la base para los cursos que impartimos a personal docente e investigador de la ULL, dentro del Plan de Formación del Profesorado, aunque en estos casos se incide mucho más en la búsqueda de información electrónica y en cómo utilizar los indicadores de calidad para los sexenios y complementos de investigación.

Otra línea de trabajo que incluye nuestro Plan de Formación son cursos de formación en competencias informacionales diseñados para el Personal de Administración y Servicios, en un primer lugar dirigidos al personal de la misma Biblioteca, pero que proyectamos extender al resto de la ULL. Y por último, incluye formación a personas externas a la ULL, especialmente personal de las bibliotecas públicas canarias que deseen actualizar sus conocimientos sobre las fuentes de información.

\section{Trabajar de forma cooperativa en formación en competencias (UNICI2)}

\subsection{Trabajar en competencias con otras bibliotecas universitarias}

Trabajar de forma cooperativa es un hecho que desde hace tiempo las bibliotecas hemos venido haciendo para diferentes áreas; en estos momentos otro de los temas que se ha añadido a la larga lista de actividades en las que cooperamos es la formación en competencias.

Al trabajar juntas varias bibliotecas universitarias hemos sido capaces de aportar ideas que hacen que el producto inicial, el modelo de curso virtual para alumnos de primer curso de grado, se enriquezca, como expondremos a continuación.

¿Qué nos decidió a compartir nuestro modelo de formación?

Entre el año 2007 al 2010, una vez habíamos implementado el modelo de curso para alumnos de primer curso en nuestra Universidad, recibimos la invitación de varias bibliotecas universitarias para que presentáramos nuestro modelo y los resultados obtenidos. En ese recorrido, que comenzó por la Biblioteca de la Universidad de Cádiz, y continuó por las de la Carlos III, Granada, Burgos, Castilla-La Mancha, Murcia, Santiago de Compostela y Zaragoza, nos dimos cuenta de que todos teníamos los mismos problemas para comenzar a implantar cursos de formación en competencias, entre otros:

- Cómo animar a los bibliotecarios para que adquirieran nuevas habilidades y reciclaran otras. 
- Cómo contar con el apoyo de la institución.

- Cómo contar con el profesorado para que apoyara el nuevo tipo de curso.

- Cómo elaborar contenidos, tareas, etcétera.

En las presentaciones que ofrecimos en las diferentes bibliotecas universitarias tratamos los miedos, los retos y las oportunidades relacionados con el nuevo modelo formativo. Aportamos nuestra experiencia y ofrecimos la posibilidad de reducir esfuerzos, evitando que cada biblioteca confeccionara el mismo curso partiendo de cero, pues los objetivos estaban claros y eran compartidos.

De la comunicación que surgió tras estas presentaciones surgió la idea de compartir el modelo de curso en competencias informacionales.

La aceptación recibida en nuestro entorno profesional se materializó en la figura de un convenio de colaboración.

\subsection{El Convenio UNICI2}

En junio de 2010 se firma un Convenio entre las universidades de La Laguna, Santiago de Compostela y Zaragoza para el desarrollo de un modelo de curso en competencias informacionales para el grado en el ámbito de las bibliotecas universitarias (2010). Posteriormente se hacen adendas al convenio, en virtud de las cuales se suman las Universidades de Alicante y Huelva. Actualmente hay varias bibliotecas pendientes de confirmar su inclusión en la adenda.

El convenio se dotó de logo y marca, UNICI2, como símbolo de unión de las principales competencias que pretendemos trabajar de forma conjunta. Por el momento la mayor parte de las bibliotecas integrantes están trabajando en competencias informacionales. El objetivo a medio plazo es poder trabajarlas conjuntamente con las informáticas.

FIGURA 2

Logo de la UNICI2

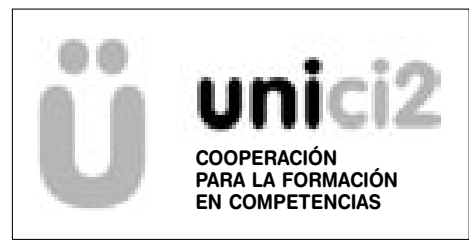

El objetivo es desarrollar un modelo común de curso de iniciación sobre competencias informacionales para alumnos de primero de grado, ante el reto del Espacio Europeo de Educación Superior y el cambio de concepción del modelo de enseñanza/aprendizaje, con el fin de mejorar la oferta formativa de las bi- 
bliotecas integrantes y evaluar de forma común el aprendizaje de los alumnos en las diferentes universidades.

Se crea una red de trabajo cooperativo, con la adopción y puesta en marcha en las universidades firmantes del programa creado por la Universidad de La Laguna, destacando en la agenda la voluntad de definir y avanzar en un plan común de investigación en competencias informacionales, producir estudios cooperativos e integrados en esta red, con datos comparables, promover esta formación e intercambiar experiencias que se puedan externalizar como ejemplo de buenas prácticas.

El convenio recoge la creación de un grupo de mejora interuniversitario. Dicho grupo trabaja de forma remota a través de un espacio creado en el Campus Virtual de la ULL y de forma presencial mediante reuniones periódicas.

El trabajo cooperativo ha sido muy fructífero. Los primeros resultados han sido expuestos en 2011 en las IX Jornadas CRAI (Santiago de Compostela), en los XVI Encuentros Internacionales sobre Sistemas de Información y Documentación (IBERSID) y en Red Telescopi, como ejemplo de buena práctica (Proyecto de cooperación interuniversitaria para el desarrollo de un curso en competencias informacionales para alumnos/as de primer grado, 2011).

Actualmente se está preparando un trabajo para presentar en el marco de INFO 2012 (La Habana), en el que además se participará en un taller de buenas prácticas ALFIN de la comunidad iberoamericana (http://www.congreso-info.cu/).

La firma del convenio se vio como una oportunidad para todos los integrantes: nuestra Biblioteca compartía un modelo, que sería evaluado y mejorado por todas las bibliotecas integrantes, con lo que se evitaba duplicar esfuerzos y se propiciaba la comparación de resultados entre un número muy significativo de alumnos.

El convenio ha supuesto una nueva forma de organización cooperativa y mayor visibilidad al proyecto.

Las bibliotecas integrantes del convenio evalúan dos aspectos fundamentales:

- El grado de aprendizaje de los alumnos en el curso.

- El grado de satisfacción de los alumnos, de los profesores colaboradores y el de los bibliotecarios formadores. Este elemento ayuda a mejorar el enfoque y los contenidos del curso adaptándolos a las necesidades de docentes y discentes.

Anualmente se recogen los datos de todas las bibliotecas que nos permiten establecer propuestas de mejora.

Los datos globales sobre la formación desarrollada en el curso académico 2010-2011 son los siguientes:

- 6.243 alumnos han realizado el curso.

- El 88\% de los alumnos lo han superado con éxito.

- 74 bibliotecarios han participado en el proyecto.

- El 85\% de los alumnos dice que ha adquirido nuevos conocimientos. 
- El 80\% de los alumnos creen que el curso les servirá en su formación académica.

- El 82\% de los alumnos recomendarían el curso a otros estudiantes.

Cada biblioteca ha asumido la coordinación de un aspecto para la mejora del modelo:

- La Laguna es responsable de los contenidos del curso. Para ver el modelo, acceder a la siguiente dirección (entrando como "invitado"): http://campusvirtual.ull.es/formacion/course/view.php?id=551

- Santiago, de la difusión interna y externa. Se ha abierto página en Facebook y en Linkedin, y enviado mensajes a los principales foros nacionales e internacionales.

- Zaragoza, de la evaluación conjunta, que ha quedado reflejada en todos los trabajos presentados por el UNICI2.

- Alicante, de los materiales didácticos: un vídeo sobre CI2 (http://hdl.handle.net/10045/18533) y un tutorial sobre cómo buscar en Internet (http://hdl.handle.net/10045/18534).

- Huelva, del estudio para lograr la certificación del curso por una agencia externa.

TABLA IV

Datos de las universidades integrandes del convenio, curso 2010-2011

\begin{tabular}{l|c|c|c|c}
\hline \multicolumn{1}{c|}{ Universidad } & Profesores & Alumnos & Grados & $\begin{array}{c}\text { Alumnos } \\
\text { de nuevo ingreso }\end{array}$ \\
\hline Alicante & 2.387 & 29.755 & 39 & 6.782 \\
\hline La Laguna & 1.716 & 24.744 & 41 & 5.053 \\
\hline Santiago & 2.302 & 29.305 & 48 & 4.508 \\
\hline Zaragoza & 3.715 & 30.478 & 54 & 7.683 \\
\hline
\end{tabular}

Las bibliotecas que se incorporen al convenio en el futuro deberán cumplir unos compromisos mínimos:

- Impartir el curso virtual sobre competencias informacionales con unos contenidos mínimos.

- Poner a disposición del resto una copia del curso que imparta, con las especificidades incorporadas.

- Compartir cualquier desarrollo o mejora del curso, ya sea en cuanto a contenidos, tareas, materiales, etcétera.

- Poner a disposición de los demás miembros los datos estadísticos de la actividad desarrollada. 
Las señas de identidad del Convenio han quedado recogidas en el artículo Trabajando juntos: hacia un modelo común de evaluación de la formación en competencias informacionales (2011):

- Es una alianza, porque hemos establecido unos acuerdos de colaboración que se recogen en el convenio.

- Es una realidad, porque hablamos de experiencias concretas, con datos comparables.

- Es un modelo formativo específico basado en la igualdad de oportunidades para todos los alumnos de nuevo ingreso.

- Es un modelo común, pero teniendo en cuenta las especificidades de cada biblioteca.

- Es un modelo evaluado de forma conjunta, compartiendo indicadores y criterios comunes.

- Es un modelo apoyado por las diferentes universidades, que ha propiciado el trabajo con profesores, coordinadores de calidad o grado y vicerrectores responsables de innovación educativa y servicios.

- Es exportable, ya que UNICI2 está difundiendo su modelo de buenas prácticas a otras bibliotecas, tanto españolas como iberoamericanas.

\section{El futuro de CI2 en las bibliotecas universitarias: conclusiones}

Cuando hablamos con compañeros de otras bibliotecas universitarias para conocer la realidad en sus universidades, vemos que todavía queda mucho camino por recorrer en relación al tema que nos ocupa.

Una buena parte de la universidad sigue estando más preocupada por implantar nuevas titulaciones, adoptar estándares de calidad e innovación, etc., que la prolija normativa educativa recoge, que de trabajar en cómo garantizar que los estudiantes adquieran unas competencias básicas transversales. La universidad está todavía asimilando muchos cambios para converger.

El futuro de CI2 será positivo si en nuestras universidades trabajamos juntos las bibliotecas, los servicios TIC y los vicerrectorados implicados en innovación educativa, alumnado y profesorado.

Cuando las universidades hayan asumido realmente en sus programas de gobierno y planes estratégicos que los alumnos deben trabajar ambas competencias, el papel de la biblioteca en este proceso, de forma conjunta con otros servicios, podrá ser una realidad. Aunque todavía nuestras universidades están lejos de las acciones que se proponen en Webber y Johnston (Pasadas, 2006) no debemos perder la esperanza de conseguirlas.

REBIUN es actualmente un gran referente, y no sólo para las bibliotecas universitarias. Como sectorial de la CRUE, ha ido a la vanguardia en nuestras uni- 
versidades en el tema de la adquisición de competencias. En su III Plan estratégico, REBIUN 2020, tiene como objetivo de su Línea 2 "Integrar de forma progresiva las Competencias Informáticas e Informacionales (CI2) en los diferentes estudios de la universidad como estrategia educativa para el desarrollo de las capacidades válidas para toda la vida” (REBIUN, 2011, p. 18).

En esa preocupación por aunar las dos competencias, está trabajando de forma conjunta y activa con otra sectorial, CRUE-TIC. CRUE-TIC y REBIUN tienen como objetivo la incorporación de las competencias transversales informáticas e informacionales en las universidades españolas. Ambas han creado ya varios productos (vídeos y documentos) muy interesantes para difundir la importancia de estas competencias y cómo se deben integrar en los estudios de grado (Comisión Mixta CRUE-TIC y REBIUN, 2009).

Las jornadas CRAI que organiza REBIUN, y últimamente las dos sectoriales, son un claro ejemplo de cooperación para trabajar CI2 en nuestras universidades. Incluso se ha llegado más lejos en los objetivos y se trabaja en la certificación de las competencias.

A pesar del esfuerzo de REBIUN, muchas bibliotecas no han visto todavía la oportunidad que el EEES trae consigo para un servicio que gestiona información. Al contemplar que los estudiantes adquieran tanto competencias informacionales como informáticas, crea un entorno favorable para que las bibliotecas mejoren su posicionamiento en su universidad. Además, si se trabaja de forma conjunta con otros servicios, para apoyar el aprendizaje, se generan sinergias que enriquecen las competencias de los bibliotecarios. Por ello, es necesario seguir haciendo esfuerzos de difusión de proyectos de bibliotecas en relación a este tema.

Quedan muchos temas pendientes para lograr una implementación real de CI2 en nuestras universidades, entre los cuales se encuentran:

- La integración en las guías docentes de las dos competencias, ya que aparecen recogidas en los títulos (una o ambas) pero no hay una plasmación real en las guías de las asignaturas.

- Inercia imperante en algunos colectivos, tanto bibliotecarios como profesores, a los que les cuesta asumir el cambio.

- Resolver el tema de la masificación de alumnos en algunas universidades, lo que dificulta que todos reciban formación en competencias CI2 de forma secuenciada.

- La despreocupación por tener evidencias del aprendizaje en competencias de los alumnos: ¿qué competencias informacionales e informáticas ha adquirido y cómo se han evaluado?

- La biblioteca como servicio de apoyo debe contar con profesionales con una carga de trabajo importante orientada a la formación en competencias. La universidad debe contar con estos aliados que son gestores de información y capacitados para reciclarse y asumir nuevos roles. 
- La biblioteca debe ser proactiva y presentar proyectos de mejora e innovación, como el de la formación en competencias en el nuevo modelo educativo. Los bibliotecarios tienen nuevas posibilidades profesionales si se actúa de forma anticipada.

- La biblioteca debe apoyar a su institución en la búsqueda de un modelo de certificación para su alumnado, como garantía del aprendizaje que ha adquirido en CI2 en su universidad.

\section{Bibliografía}

CI2: competencias informacionales e informáticas (2011). Disponible en: http://ci2.es/ [consulta: 31 de enero de 2012].

Comisión Mixta CRUE-TIC y REBIUN (2009): Competencias informáticas e informacionales en los estudios de grado [en línea]. Disponible en: http://crue-tic.uji.es/index.php?option $=$ com_remository\&Itemid $=28 \&$ func $=$ startdown\&id $=226$ [consulta: 31 de enero de 2012].

Competencias informacionales y digitales en educación superior (2010). Coordinado por Manuel Area Moreira. Monográfico de RUSC. Revista de Universidad y Sociedad del Conocimiento, 7(2). Disponible en: http://rusc.uoc.edu/ojs/index.php/rusc/article/ view/v7n2-area [consulta: 13 de febrero de 2012].

Convenio entre las universidades de La Laguna, Santiago de Compostela y Zaragoza para el desarrollo de un modelo de curso en competencias informacionales para el grado en el ámbito de las bibliotecas universitarias (2010). Disponible en: https://docs.google. $\mathrm{com} /$ viewer? $\mathrm{a}=\mathrm{v} \& \mathrm{pid}=$ sites\&srcid=dWxsLmVkdS5lc3x1bmljaTJ8Z3g6NzkyMTk4YjYwZTExYjg3Yg [consulta: 20 de marzo de 2012].

González, N.; Hernández, C. J. (2007). Nuevos roles del bibliotecario/documentalista. XII Jornadas Nacionales de Información y Documentación en Ciencias de la Salud. Disponible en: http://www.slideshare.net/nievesglez/nuevos-roles-del-bibliotecario [consulta: 20 de enero de 2012].

Hernández, C. J. (2010). Un plan de formación en competencias de información a través de aulas virtuales: análisis de una experiencia con alumnado universitario. Revista de Universidad y Sociedad del Conocimiento, vol. 7, n. ${ }^{\circ}$ 2. Disponible en: http://rusc. uoc.edu/ojs/index.php/rusc/issue/view/v7n2 [consulta: 20 de enero de 2011].

Hernández, C. J. (2011). La experiencia de la Biblioteca de la ULL en formación en competencias. Veintintantas experiencias ALFIN y una canción esperanzada. Disponible en: http://www.alfared.org/content/veintitantas-experiencias-alfin-y-una-canci-n-esperanzada/1287 [consulta: 31 de enero de 2012].

Jornadas CRAI (9. ', Santiago de Compostela, 2011). Disponible en: http://www.usc.es/es/ congresos/crai/post.html [consulta: 31 enero 2012].

Pasadas, C. (2006). Hacia una universidad alfabetizada en información según Sheila Webber y Bill Johnston. Boletín de la Asociación Andaluza de Bibliotecarios, n. ${ }^{\circ}$ 84-85, pp. 47-52. Disponible en: http://www.aab.es/pdfs/baab84-85/84-85a4.pdf [consulta: 12 de febrero de 2012]. 
Proyecto de cooperación interuniversitaria para el desarrollo de un curso en competencias informacionales para alumnos/as de 1.er de grado (2001). Disponible en: http://telescopi.upc.edu/bdcasos/?page=bp_ver\&tipo=\&area $=3 \&$ keyword $=\& p a i s=0 \& p a$ gina $=1 \&$ id=155 [consulta: 31 de enero de 2012].

REBIUN (2011). III Plan Estratégico de REBIUN 2020. Disponible en: http://rebiun.org/ opencms/opencms/handle404?exporturi=/export/docReb/PE_REBIUN_2020.pdf\&\%5d [consulta: 31 de enero de 2012].

Trabajando juntos: hacia un modelo común de evaluación de la formación de competencias informacionales (2011). Grupo de trabajo UNICI2. Jornadas CRAI (9. ${ }^{\circ}$, Santiago de Compostela, 2011). Disponible en: http://www.slideshare.net/CarmenVarelaPrado/uni-ci2-cooperacion-para-la-formacion-en-competencias-modo-de-compatibilidad-8 423699 [consulta: 11 de diciembre de 2011].

Universidad de La Laguna (2011). Plan de Gobierno 2011-2015. 58 p. Disponible en: http://www.ull.es/Private/folder/institucional/ull/wull/destacados/plan_gobierno 201115.pdf [consulta: 31 de enero de 2012]. 